\title{
Photoelectrochemical water splitting: silicon photocathodes for hydrogen evolution
}

\author{
Emily L. Warren*, Shannon W. Boettcher, James R. McKone, Nathan S. Lewis \\ California Institute of Technology, 1200 E. California Blvd, Pasadena, CA, USA 91125
}

\begin{abstract}
The development of low cost, scalable, renewable energy technologies is one of today's most pressing scientific challenges. We report on progress towards the development of a photoelectrochemical water-splitting system that will use sunlight and water as the inputs to produce renewable hydrogen with oxygen as a by-product. This system is based on the design principle of incorporating two separate, photosensitive inorganic semiconductor/liquid junctions to collectively generate the 1.7-1.9 $\mathrm{V}$ at open circuit needed to support both the oxidation $\mathrm{H}_{2} \mathrm{O}$ (or $\mathrm{OH}^{-}$) and the reduction of $\mathrm{H}^{+}$(or $\mathrm{H}_{2} \mathrm{O}$ ). Si microwire arrays are a promising photocathode material because the high aspect-ratio electrode architecture allows for the use of low cost, earth-abundant materials without sacrificing energy-conversion efficiency, due to the orthogonalization of light absorption and charge-carrier collection. Additionally, the high surfacearea design of the rod-based semiconductor array inherently lowers the flux of charge carriers over the rod array surface relative to the projected geometric surface of the photoelectrode, thus lowering the photocurrent density at the solid/liquid junction and thereby relaxing the demands on the activity (and cost) of any electrocatalysts. Arrays of $\mathrm{Si}$ microwires grown using the Vapor Liquid Solid (VLS) mechanism have been shown to have desirable electronic light absorption properties. We have demonstrated that these arrays can be coated with earth-abundant metallic catalysts and used for photoelectrochemical production of hydrogen. This development is a step towards the demonstration of a complete artificial photosynthetic system, composed of only inexpensive, earth-abundant materials, that is simultaneously efficient, durable, and scalable.
\end{abstract}

Keywords: hydrogen, water-splitting, solar, silicon, photocathode

\section{INTRODUCTION}

\subsection{Solar Water Splitting}

Solar energy is the only renewable energy source with the capacity to completely satisfy the projected energy consumption of our planet over the next century. ${ }^{1}$ While there are several ways to harness sunlight to do useful work, the diurnal cycle necessitates the storage of solar energy if it is going to compete with existing base load technologies such as coal-fired electricity and nuclear power plants. The development of a stable and efficient materials system that can store the energy in sunlight by splitting water into hydrogen and oxygen has been considered for decades as one of the "Holy Grails" of science and technology. ${ }^{2}$ To create a fully integrated water splitting device, the principles of semiconductor physics must be combined with those of electrocatalysis. The unassisted photoelectrolysis of water requires $1.23 \mathrm{~V}$ of photopotential from semiconducting materials that have band edges which span both the oxidation and reduction potentials for the decomposition of water. No known stable semiconductor with the appropriate band energetics is capable of producing significant photocurrent for this reaction.

One approach to circumvent this problem is to use two semiconductors, with complimentary absorption characteristics, in series and to separately optimize the photocathode and photoanode for the reduction and oxidation reactions, respectively. The theoretical efficiency for such a device, with a photoanode material with a band gap of $1.7 \mathrm{eV}$ and a photocathode material with a bandgap of $1.1 \mathrm{eV}$, is $27.9 \%{ }^{3}$ Such a system requires four photons to produce one molecule of $\mathrm{H}_{2}$, paralleling energy requirements for biological photosynthesis.

\footnotetext{
*Email: ewarren@caltech.edu; phone 626-395-3964; www.nsl.caltech.edu
}

Solar Hydrogen and Nanotechnology V, edited by Hicham Idriss, Heli Wang, Proc. of SPIE Vol. 7770, 77701F · ( 2010 SPIE · CCC code: 0277-786X/10/\$18 · doi: 10.1117/12.860994 


\subsection{Water splitting cell design}

While several attempts have been described in the literature to design an unassisted photoelectrolysis cell, the most efficient devices have been too expensive to be implemented at the global scale. ${ }^{4}$ We have proposed a device that can be manufactured cheaply and that is theoretically capable of splitting water with no external bias. The schematic shown in Figure 1 consists of earth-abundant photoanode and photocathode materials, separated by a proton-permeable membrane. The photoanode and photocathode will consist of rod-like semiconductor components, with attached heterogeneous multi-electron transfer catalysts, that are needed to drive the oxidation or reduction reactions at low overpotentials. The high aspect-ratio semiconductor rod electrode architecture allows for the use of low cost, earthabundant materials without sacrificing energy conversion efficiency, due to the orthogonalization of light absorption and charge-carrier collection. Additionally, the high surface-area design of the rod-based semiconductor array inherently lowers the flux of charge carriers over the rod array surface relative to the projected geometric surface of the photoelectrode, thus lowering the photocurrent density at the solid/liquid junction, and thereby relaxing the demands on the activity (and cost) of any electrocatalysts.

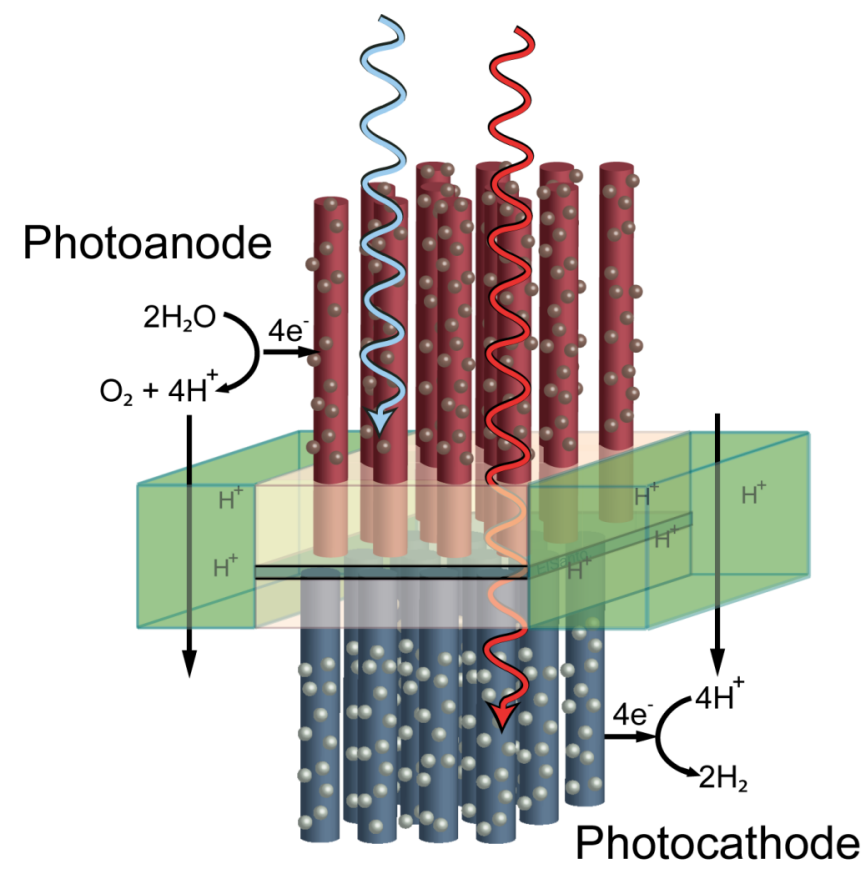

Figure 1. Schematic of the proposed artificial photosynthesis water splitting device. The photoanode (top) consists of a metal oxide semiconductor material decorated with oxygen evolving catalysts and absorbs short wavelength light. The photocathode (bottom) will consist of Si microwires decorated with a hydrogen evolution reaction (HER) catalyst. The two semiconductors are electrically connected and separated in a proton conductive membrane that separates the gas products but allows ions to pass to balance the chemical reaction.

This device design allows a parallel construction approach for each component. We have previously reported on the development of a nano-porous $\mathrm{WO}_{3}$ photoanode material with greatly improved light absorption. ${ }^{5}$ Additionally we have shown that we can use polydimethylsiloxane (PDMS) as a structural support, to create a membrane of Si microwires. ${ }^{6}$ The remainder of this paper will discuss progress that has been made on characterizing the photoelectrochemical performance of Si microwires to serve as the photocathode of this water-splitting device.

\section{SILICON PHOTOCATHODES}

Silicon is the most widely used semiconductor for photovoltaic applications. In addition to being earth abundant, and well characterized in photovoltaic systems, $\mathrm{Si}$ also has the appropriate band energetics to serve as the photocathode for a water-splitting cell (Figure 2). The Si surface, however, has poor kinetics for the hydrogen evolution reaction (HER), 
and requires a catalyst to achieve efficient solar energy conversion. ${ }^{7}$ There are several accounts in the literature of Sibased photocathodes, but most devices have relied on noble metal catalysts such as Pt or Pd, that are too rare and expensive to be viable options for a low-cost water-splitting device. ${ }^{8}$ As mentioned above, in a wire geometry, the photocurrent will be distributed across the surface of the wires, lowering the electron flux across the junction and allowing for higher catalyst loadings. This increase in surface area will also decrease the attainable photovoltage from the system, because the dark current will increase with junction area, and this effect must be accounted for in an optimized cell design. ${ }^{9}$
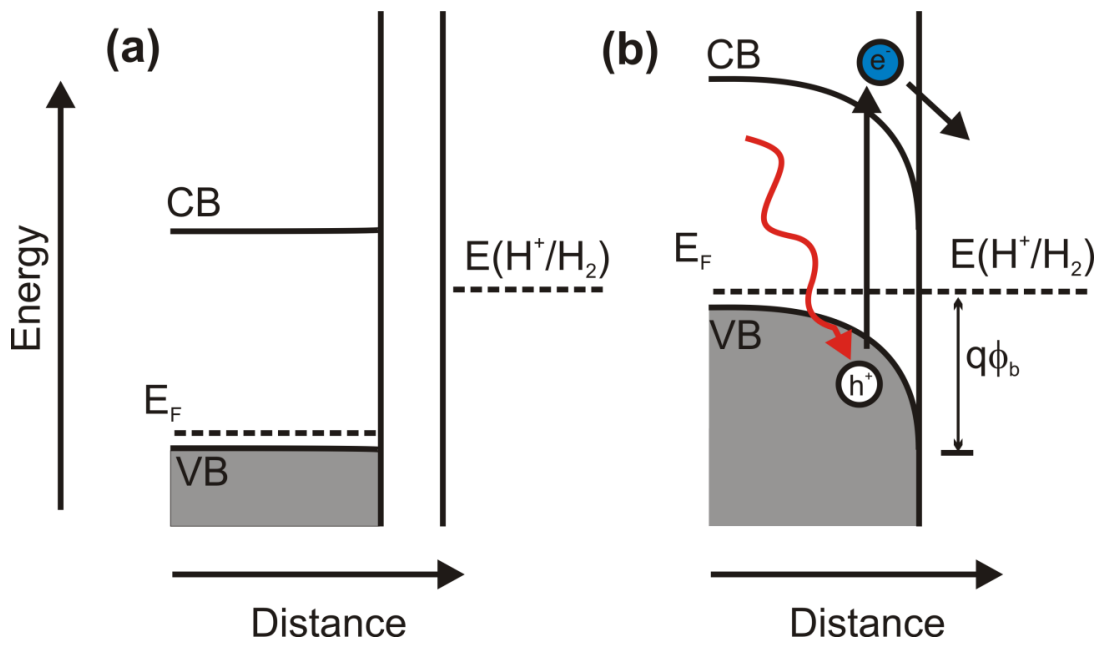

Figure 2. Band energetics of $\mathrm{p}-\mathrm{Si}$ (a) before equilibration with solution, (b) after equilibration and under illumination. The equilibration of the semiconductor Fermi level $\left(E_{F}\right)$ with the solution potential $\left(E\left(H^{+} / H_{2}\right)\right.$ creates an electric field at the interface, which separates photogenerated charges and allows them to do electrochemical work.

In traditional planar semiconductor junctions, the directions of light absorption and charge-carrier collection are the same. Hence, optically thick materials are needed to collect all of the photons, and the diffusion length of the material must be long enough to allow the photogenerated minority carriers to reach the junction. In a radial geometry, the directions of light absorption and carrier diffusion are decoupled, which allows material with lower diffusion lengths to effectively capture all of the photogenerated carriers (Figure 3). By creating an array that is thick enough to absorb the majority of the incident light, and with the wire radius on the scale of the diffusion length of the material, materials with much lower diffusion lengths can achieve efficiencies comparable to those of planar cells. ${ }^{9}$ In the case of $\mathrm{Si}$ (an indirect band-gap semiconductor) a $100 \mu \mathrm{m}$ penetration depth is needed to absorb $90 \%$ of the above bandgap solar spectrum.

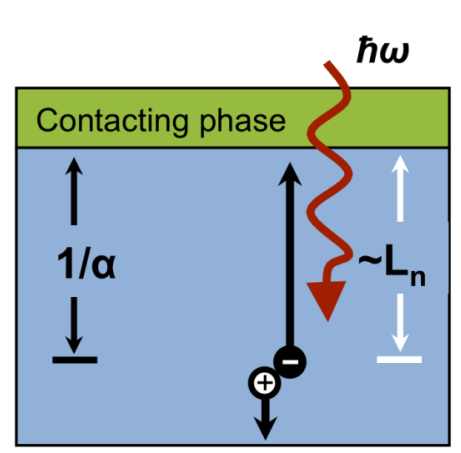

(a) Si wafer

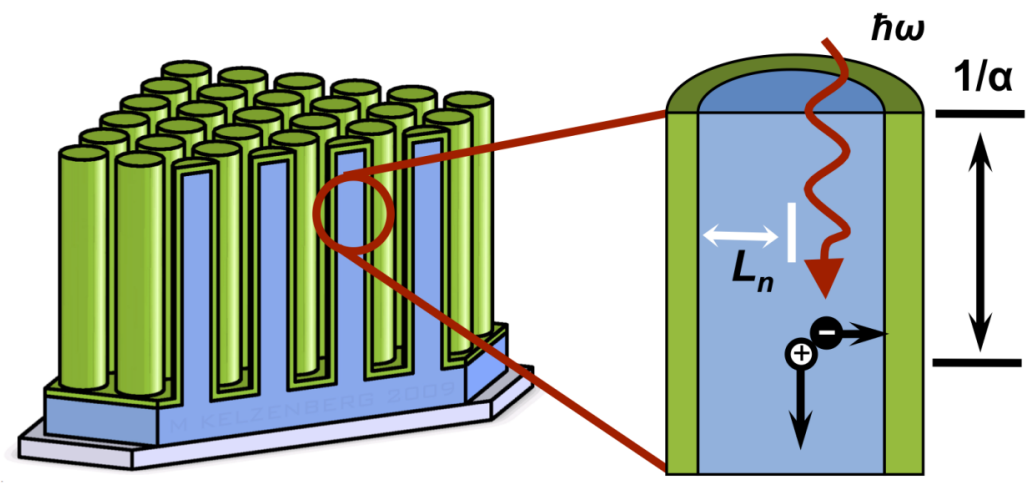

(b) Si wire array

Figure 3. Comparison of a planar (a) and radial (b) Si photoconversion system. In the former, the minority-carrier diffusion length must scale with the absorption length of a photon, to capture the photogenerated carrier before it recombines. In a radial geometry, these two phenomena are decoupled, and any photogenerated carrier that can traverse the radius of the wire will be collected. 


\subsection{Fabrication of Si microwire arrays}

We have developed a process to grow arrays of Si microwires over large areas $\left(>1 \mathrm{~cm}^{2}\right)$ using the vapor-liquid-solid (VLS) mechanism. ${ }^{10,11}$ Boron-doped $\mathrm{p}^{+}-\mathrm{Si}(111)$ wafers coated with $300-600 \mathrm{~nm}$ of thermal oxide were patterned with holes that were defined in the oxide by photolithographic exposure and development of a photoresist layer (Microchem S1813), followed by a buffered HF(aq) (BHF) etch. The size of the holes can be controlled by choice of the photolithographic mask. The copper VLS catalyst was then deposited via thermal evaporation onto the patterned photoresist, followed by liftoff of the photoresist and excess metal. Substrates were then annealed in a tube furnace for 20 min at $1000{ }^{\circ} \mathrm{C}$ under $\mathrm{H}_{2}$. Wires were grown and doped p-type by flowing $\mathrm{SiCl}_{4}, \mathrm{BCl}_{3}\left(0.25 \%\right.$ in $\left.\mathrm{H}_{2}\right)$, and $\mathrm{H}_{2}$ at rates of 10, 1.0, and $500 \mathrm{sccm}$, respectively, for 20-30 min. After growth, the tube was purged with $\mathrm{N}_{2}$ and allowed cool to $650{ }^{\circ} \mathrm{C}$ over the course of $\sim 30 \mathrm{~min}$. The resulting wires were typically between $40-80 \mu \mathrm{m}$ in length and $1-2 \mu \mathrm{m}$ in diameter. These growth conditions have been shown to yield p-Si wires with active doping concentrations of $\sim 10^{17} \mathrm{~cm}^{-}$ ${ }^{3.12}$ The $\mathrm{Cu}$ VLS catalyst was removed by etching the wires with an RCA2 clean $\left(6: 1: 1 \mathrm{H}_{2} \mathrm{O}: \mathrm{HCl}: \mathrm{H}_{2} \mathrm{O}_{2}\right.$ at $70{ }^{\circ} \mathrm{C}$ for 15 $\min )$.

\subsection{Material characterization}

Before testing the wire arrays for their hydrogen production capabilities, the electronic properties of the wires were investigated using a one-electron outer-sphere redox couple in an aqueous environment. These experiments decouple the semiconductor performance of the wire arrays from the more complicated chemistry of water splitting. Methyl viologen $\left(\mathrm{MV}^{2+/}\right)$ is a well-documented redox couple that creates a high barrier height to $\mathrm{p}-\mathrm{Si}^{13}$ The current density vs. potential properties of the wires were tested by making electrical contact to the Si substrate with $\mathrm{Ga} / \mathrm{In}$, and then encapsulating the wires with epoxy (Hysol $1 \mathrm{C}$ ). Electrochemistry was performed using $50 \mathrm{mM}$ of $\mathrm{MV}^{2+/+}$ in a pH 2.9 phthalate buffer with $0.5 \mathrm{M}$ of $\mathrm{K}_{2} \mathrm{SO}_{4}$ as supporting electrolyte. An $808 \mathrm{~nm}$ monochromatic light source was used to minimize the optical absorption of the $\mathrm{MV}^{+}$radical cation.
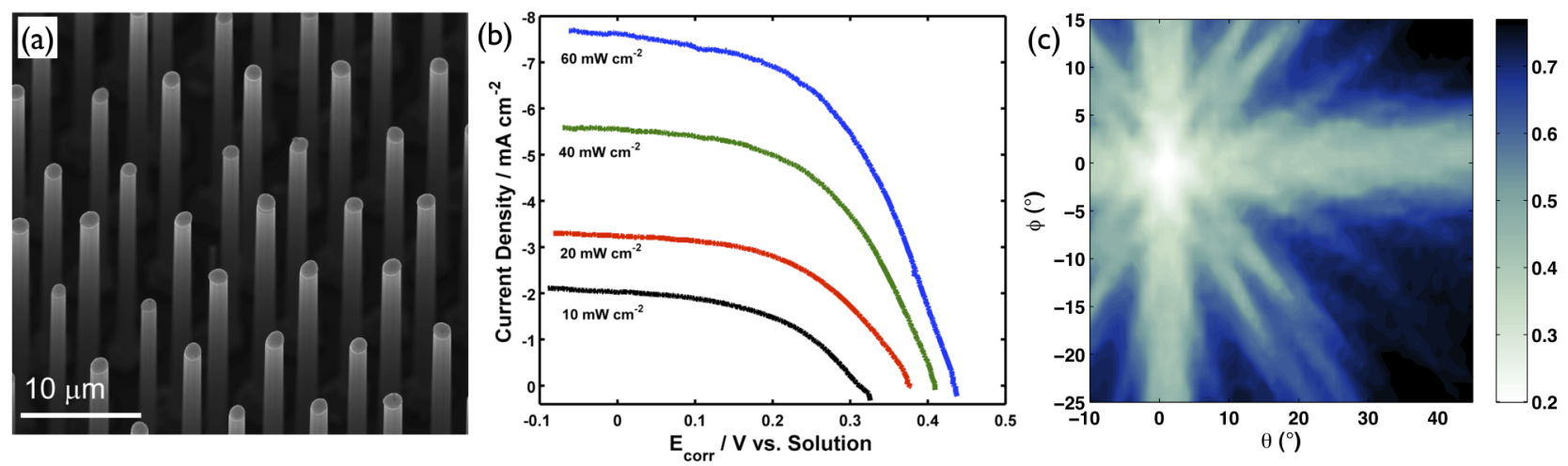

Figure 4. (a) SEM image of Si microwires, (b) Current density vs. potential data for a representative Si microwire array in $\mathrm{MV}^{2+++}\left(E_{\text {sol }}=-0.60 \mathrm{~V}\right.$ vs a saturated calomel electrode (SCE)) at a variety of illumination intensities, (c) Angle dependent external quantum yield data. The data in (b) were taken at $(\theta, \phi=0)$.

Figure $4 \mathrm{~b}$ shows the device performance of a representative wire arrays. Under $60 \mathrm{~mW} \mathrm{~cm}$ at $808 \mathrm{~nm}$ illumination at normal incidence $(\theta, \phi=0)$ the wire arrays exhibited open-circuit voltages $\left(V_{o c}\right)$ of $0.41 \pm 0.04 \mathrm{~V}$, short-circuit current densities $\left(J_{s c}\right)$ of $7.7 \pm 0.9 \mathrm{~mA} \mathrm{~cm}{ }^{-2}$, and fill factors $(f f)$ of $0.50 \pm 0.10$ after correcting for concentration over-potential and uncompensated cell resistances. ${ }^{13}$ For comparison, planar Czochralski-grown Si wafers run in the same cell exhibited $V_{o c}=0.55 \pm 0.015 \mathrm{~V}, J_{s c}=27.9 \pm 0.8 \mathrm{~mA} \mathrm{~cm}{ }^{-2}, f f=0.51 \pm 0.05$. The wire-array device characteristics produced an overall conversion efficiency $\left(\eta_{808}\right)$ of $2.6 \pm 0.4 \%$ for the wire arrays and $12.0 \pm 1.0 \%$ for the planar control. ${ }^{13}$ These results demonstrate that photogenerated carriers can be efficiently collected from these wires in a liquid-junction cell. The significantly lower current densities of the wire samples can be attributed to the fact that the wires have approximately $1 \%$ as much photoactive Si as the planar samples. A recent absorption study on Si wire arrays has shown a large angular dependence of the absorption of the wire arrays. ${ }^{14}$ Using the same electrochemical system as described above, but with a 
focused beam light source (so all photons hit the sample) the angle of incidence on the wires can be controlled, and the changes in external quantum efficiency can be tracked. The EQE increased significantly at higher angles of incidence when the photons had a longer path length within the wire array (Figure 4c). This behavior demonstrates that increasing the light absorption in the wires can increase the photocurrent and photovoltage attainable from the wires. By incorporating highly scattering nanoparticles, and by addition of a back contact to the wire array, the overall light absorbance has been increased, and the angular dependence of these wires decreased still further. ${ }^{14}$

\subsection{Catalyst attachment}

Prior work on p-Si photocathode systems has clearly demonstrated that a catalyst is required to produce hydrogen at potentials positive of the thermodynamic reduction potential. ${ }^{7}$ Many reports are in the literature on the catalytic activity of sub-monolayers of metals on p-Si surfaces. ${ }^{15}$ Due to the differences in light absorption and diffusion within an array of Si wires relative to the planar samples, the deposition conditions must be controlled carefully to optimize the particle size and uniformity for the high aspect-ratio wires. Uniform distributions of metal nanoparticle films were produced by using galvanic displacement deposition, in which metal ions from solution are reduced as the Si is slowly etched by hydrofluoric acid (HF) without any external bias. ${ }^{16}$ By modifying the deposition procedures from the literature, we were able to achieve uniform distribution of nanoparticles of several metals that have high HER exchange current densities on the high aspect ratio Si microwires. Pt nanoparticles were deposited from an aqueous solution of hydrofluoric acid (HF) and chloroplatinic acid $\left(\mathrm{H}_{2} \mathrm{PtCl}_{6}\right){ }^{8}$ Decreasing the concentration of the $\mathrm{Pt}$ salt increased the uniformity of deposition across the length of the wires. Ni nanoparticles were deposited from an aqueous solution of ammonium fluoride $\left(\mathrm{NH}_{4} \mathrm{~F}\right)$ and nickel sulfate $\left(\mathrm{NiSO}_{4}\right)$, and also uniformly covered the surfaces of the wires. ${ }^{17}$
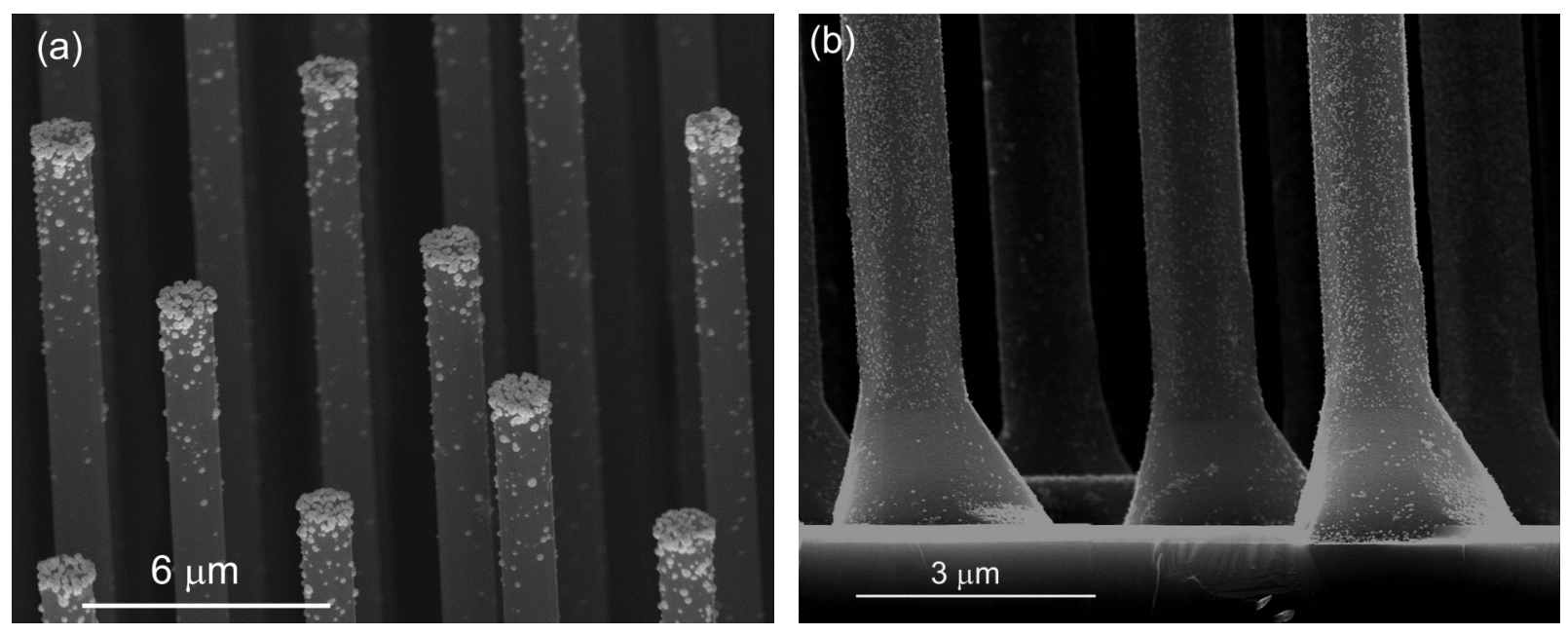

Figure 5. SEM image of wires with metal nanoparticle catalysts, (a) Pt on Si microwires. (b) Ni on Si microwires.

\subsection{Comparing catalyst performance}

The catalytic enhancement of the HER by metal nanoparticle films was investigated on planar Si electrodes. Photoelectrochemical measurements were performed in three-electrode cell in which a saturated calomel (SCE) electrode was used as the reference electrode and a Pt mesh served as the counter electrode. The samples were illuminated with an ELH lamp that approximated the solar spectrum. The electrolyte consisted of a pH 6.2 phosphate buffer to improve the stability of the Ni nanoparticles that are not stable under acidic conditions (where most literature reports of HER electrochemistry take place). The solution was continuously purged with $\mathrm{H}_{2}$ so that both forms of the redox couple $\left(\mathrm{H}^{+}\right.$and $\left.\mathrm{H}_{2}\right)$ were present in solution, to create a defined Nernstian solution potential. Electrodes were fabricated as in section 2.2 and dipped in HF before performing electrochemistry. After a control run without catalysts, the electrodes were immersed in the galvanic plating solutions for 1-5 min, rinsed with $\mathrm{H}_{2} \mathrm{O}$, dried with $\mathrm{N}_{2}$, and then retested. 


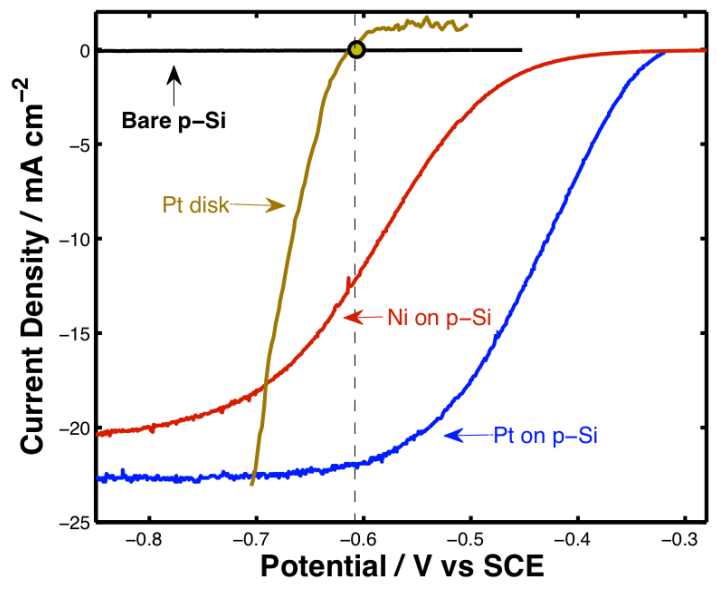

Figure 6. Comparison of $\mathrm{Ni}$ and $\mathrm{Pt}$ nanoparticles on planar Si photocathodes in $\mathrm{pH} 6.2$ phosphate buffer. All samples were illuminated with $100 \mathrm{~mW} \mathrm{~cm}^{-2}$ ELH illumination and the system was continuously purged with $\mathrm{H}_{2}$. The black curve is bare p-Si, the yellow curve is a Pt disk, the blue curve is Pt nanoparticles on p-Si, and the red curve is Ni nanoparticles on $\mathrm{p}-\mathrm{Si}$. Both the $\mathrm{Ni}$ - and Pt-decorated electrodes passed cathodic currents at potentials positive of the thermodynamic hydrogen potential in the solution (indicated by dashed line) meaning .

The Nernst equation predicts that the thermodynamic $\mathrm{H}^{+} / \mathrm{H}_{2}$ reduction potential is $-0.608 \mathrm{~V}$ vs. $\mathrm{SCE}$ at this $\mathrm{pH}$, and this was confirmed by the turn-on potential of a polished Pt electrode (Figure 6). Without any catalyst on the surface, the $\mathrm{Si}$ electrodes required over $400 \mathrm{mV}$ of over-potential before they passed any significant cathodic current. Addition of Pt nanoparticles to the surface shifted the onset of $\mathrm{H}_{2}$ production significantly, to $\sim 300 \mathrm{mV}$ positive of the thermodynamic hydrogen potential, which is indicated by the dotted line in Figure 6. The catalyst removes the kinetic barrier to passing charge across the semiconductor/liquid contact and allows the photogenerated carriers to drive the reduction reaction. When the same experiments were performed with the Ni catalysts, a slight increase in photovoltage was observed relative to Pt, but more significantly, the slower kinetics of $\mathrm{Ni}$ as a catalyst greatly decreased the attainable current at potentials positive of the reversible hydrogen potential. These results confirm that our nanoparticle catalysts allow us to carry out the HER, and preliminary studies have been conducted to understand the current density vs. potential behavior of the Si microwires decorated with same catalysts. Our preliminary results have shown hydrogen production can be obtained, albeit at low efficiencies, using p-Si microwires decorated with Pt and Ni nanoparticles. Work is currently underway to optimize this system and increase the conversion efficiency of these microwire devices using earthabundant catalysts.

\section{CONCLUSIONS}

Si microwires decorated with metal catalysts are a promising material for photocathodes for the generation of $\mathrm{H}_{2}$ from water. The Si microwires can be grown from inexpensive precursors, and their radial geometry allows the wires to effectively absorb sunlight and collect photogenerated carriers. The enhanced surface area of wire arrays relative to planar samples allows the use of earth-abundant catalysts, rather than noble metals, to drive the HER. Progress as been made on characterizing the material properties of the wire arrays, as well as characterizing their performance as $\mathrm{H}_{2}-$ evolving photocathodes. Our recent work has demonstrated that we can fabricate both the photoanode and photocathode of our proposed water-splitting device, indicating progress towards the design of an integrated solar-fuel generator.

\section{ACKNOWLEDGEMENTS}

We acknowledge Dr. Michael Kelzenberg, Dr. Josh Spurgeon, and Dr. Michael Walter for their contributions. We also thank the members of the NSF Powering the Planet Center for Chemical Innovation for their valuable contributions to this work. We gratefully acknowledge critical support and infrastructure provided for this work by the Kavli Nanoscience Institute at Caltech, the Stanford Global Climate and Energy Project and the U.S. Department of Energy (grant DE-FG02-05ER15754) for financial support. 


\section{REFERENCES}

[1] N. S. Lewis, and D. G. Nocera, "Powering the planet: Chemical challenges in solar energy utilization," Proceedings of the National Academy of Sciences of the United States of America, 104(50), 20142-20142 (2007).

[2] A. J. Bard, and M. A. Fox, “Artificial Photosynthesis: Solar Splitting of Water to Hydrogen and Oxygen," Accounts of Chemical Research, 28(3), 141-145 (1995).

[3] J. R. Bolton, S. J. Strickler, and J. S. Connolly, "Limiting and Realizable Efficiencies of Solar Photolysis of Water," Nature, 316(6028), 495-500 (1985).

[4] O. Khaselev, and J. A. Turner, "A monolithic photovoltaic-photoelectrochemical device for hydrogen production via water splitting," Science, 280(5362), 425-427 (1998).

[5] K. R. R. Gil, J. M. Spurgeon, and N. S. Lewis, "Silicon and tungsten oxide nanostructures for water splitting." Proc. SPIE 7408, 74080 S-11 (2009).

[6] K. E. Plass, M. A. Filler, J. M. Spurgeon et al., "Flexible Polymer-Embedded Si Wire Arrays," Advanced Materials, 21(3), 325-328 (2009).

[7] R. N. Dominey, N. S. Lewis, J. A. Bruce et al., "Improvement Of Photo-Electrochemical Hydrogen Generation By Surface Modification Of P-Type Silicon Semiconductor Photo-Cathodes," Journal of the American Chemical Society, 104(2), 467-482 (1982).

[8] I. Lombardi, S. Marchionna, G. Zangari et al., "Effect of Pt particle size and distribution on photoelectrochemical hydrogen evolution by pSi photocathodes," Langmuir, 23(24), 12413-12420 (2007).

[9] B. M. Kayes, H. A. Atwater, and N. S. Lewis, "Comparison of the device physics principles of planar and radial p-n junction nanorod solar cells," Journal of Applied Physics, 97(11), (2005).

[10] B. M. Kayes, M. A. Filler, M. C. Putnam et al., "Growth of vertically aligned Si wire arrays over large areas $\left(>1 \mathrm{~cm}^{2}\right)$ with $\mathrm{Au}$ and $\mathrm{Cu}$ catalysts," Applied Physics Letters, 91(10), 103110 (2007).

[11] R. S. Wagner, and W. C. Ellis, "Vapor-Liquid-Solid Mechanism Of Single Crystal Growth ( New Method Growth Catalysis From Impurity Whisker Epitaxial + Large Crystals Si E )," Applied Physics Letters, 4(5), 89-\& (1964).

[12] M. C. Putnam, D. B. Turner-Evans, M. D. Kelzenberg et al., "10 $\mu \mathrm{m}$ minority-carrier diffusion lengths in Si wires synthesized by Cucatalyzed vapor-liquid-solid growth," Applied Physics Letters, 95(16), 163116-3 (2009).

[13] S. W. Boettcher, J. M. Spurgeon, M. C. Putnam et al., "Energy-Conversion Properties of Vapor-Liquid-Solid-Grown Silicon Wire-Array Photocathodes," Science, 327(5962), 185-187 (2010).

[14] M. D. Kelzenberg, S. W. Boettcher, J. A. Petykiewicz et al., "Enhanced absorption and carrier collection in Si wire arrays for photovoltaic applications," Nature Materials, 9(3), 239-244 (2010).

[15] M. Szklarczyk, and J. Ombockris, "Photoelectrocatalysis and Electrocatalysis on P-Silicon," Journal of Physical Chemistry, 88(9), 18081815 (1984).

[16] C. Carraro, R. Maboudian, and L. Magagnin, "Metallization and nanostructuring of semiconductor surfaces by galvanic displacement processes," Surface Science Reports, 62(12), 499-525 (2007).

[17] X. Zhang, Z. Chen, and K. N. Tu, "Immersion nickel deposition on blank silicon in aqueous solution containing ammonium fluoride," Thin Solid Films, 515(11), 4696-4701 (2007). 